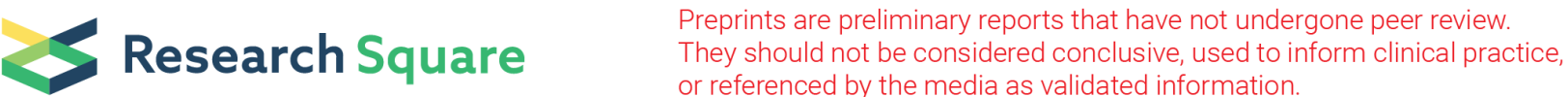

\section{Deficiency in interleukin-18 ameliorated glial activation and neuroinflammation after traumatic brain injury in mice}

\section{Wenwen Dong}

China Medical University

\section{Linlin Wang}

China Medical University

\section{Ziyuan Chen}

China Medical University

\section{Xiangshen Guo}

China Medical University

\section{Pengfei Wang}

China Medical University

\section{Hao Cheng}

China Medical University

\section{Fuyuan Zhang}

China Medical University

\section{Bei Yang}

China Medical University

\section{Shuaibo Wang}

China Medical University

\section{Ruiqun Qi}

China Medical University

Jiawen Wang

Guizhou Medical University

\section{Dawei Guan}

China Medical University

Rui Zhao ( $\nabla$ rzhao@cmu.edu.cn )

China Medical University https://orcid.org/0000-0003-4867-2503

\section{Research}

Keywords: Traumatic brain injury (TBI), Interleukin-18 (IL-18), Neuroinflammation, Microglia, Astrocyte, Cytokine, Chemokine 
Posted Date: February 25th, 2020

DOI: https://doi.org/10.21203/rs.2.24449/v1

License: (c) (i) This work is licensed under a Creative Commons Attribution 4.0 International License. Read Full License 


\section{Abstract}

Background: Neuroinflammation is recognized as one of the main pathological mechanisms of secondary injury caused by traumatic brain injury (TBI). It has been reported that interleukin (IL)-18 is expressed in glial cells and involved in the regulation of neuroinflammation. Further studies have revealed that IL-18 expression is upregulated and may contribute to pathogenesis in the later phases of TBl; however, the mechanism underlying the effect of IL-18 on TBI remains unclear. Our present study assessed the roles of IL-18 in inflammatory and neurodegenerative pathology in mice subjected to TBI.

Methods: A controlled cortical impact (CCI) injury model was conducted to mimic $\mathrm{TBI}$, and brains were collected at 3 and 7 days post TBI (dpi). The levels of IL-18 were detected by qRT-PCR and immunofluorescence staining. In addition, neurological severity score (NSS) was used to assess neurological deficits after TBI. Furthermore, neuronal cell death, glial activation, and inflammatory cytokine and chemokine secretion were evaluated in wild-type ( WT ) and II18-knockout ( II18-KO) mice to explore the role of IL-18 in TBI.

Results: IL-18 levels were upregulated post TBI, accompanied by reactive glial activation. II-18 deficiency significantly ameliorated glial activation and improved neuronal cell death and neurological deficits. In addition, II-18 deficiency reduced the TBI-induced M1-like microglia frequency. Interestingly, the levels of all pro- and anti-inflammatory cytokines, including IL-1a, IL-1 $\beta$, IL-2, IL-3, IL-4, IL-5, IL-6, IL-9, IL-10, IL12p40, IL-12p70, IL-13, IL17A, G-CSF, GM-CSF, IFN- $\gamma$, and TNF-a, were downregulated in II18 -KO mice. The deletion of II-18 attenuated the levels of most chemokines induced by TBI, including CCL2, CCL3, CCL4, CCL5, CCL7, CCL12, CCL20, CXCL1, CXCL2, CXCL10, CXCL12, CXCL13, and CXCL16.

Conclusions: These data demonstrated that IL-18 is involved in TBI induced neuroinflammation, which suggests that IL-18 is important for the development of secondary injury induced by TBI.

\section{Background}

Traumatic brain injury (TBI) is a major cause of death and disability worldwide and represents a critical public health problem, especially in China [1-3]. TBI is defined as brain damage caused by an external force that leads to an alteration in brain function or other evidence of brain pathology $[3,4]$. Both primary and secondary brain injury compose the complex pathogenesis of TBI. Neuroinflammation is recognized as one of the main pathological mechanisms of TBI-induced secondary injury $[5,6]$, which is a multifaceted response involving activation of microglia and astrocytes and production of cytokines and chemokines [5]. Brain-resident astrocytes and microglia are key players in initiating inflammatory responses post $\mathrm{TBI}$, which influence the local microenvironment and thus determine the extent of damage, by secreting various cytokines and chemokines [7]. Astrocytes can both respond to and produce many inflammatory mediators, including cytokines and chemokines [8]. Reactive astrocytes can also influence the responses of other cell types, including microglia, through cytokine production [6, 7]. After $\mathrm{TBI}$, microglia are activated, secret and respond to inflammatory mediators. Microglia become polarized 
from a resting state along an activation spectrum ranging from the classical M1-like phenotype to the alternative M2-like phenotype post TBI $[9,10]$. The different types of microglia appear to have different functions post TBI. M1-like 'proinflammatory' microglia appear to play a detrimental role, while M2-like 'anti-inflammatory' microglia show protective effects post TBI [7, 9].

Interleukin (IL)-18, initially discovered as an interferon (IFN)- $y$-inducing factor, is a member of the IL-1 family of proinflammatory cytokines. IL-18 is produced as a biologically inactive precursor protein (pro-IL18 ) that is subsequently processed by caspase- 1 into its mature and active form [11-13]. Under inflammatory conditions, activated IL-18 binds to its heterocomplex IL-18 receptor (IL-18R), which leads to activation of the transcription factor NF-KB via a complex intracellular signaling cascade, culminating in the production and release of several cytokines, chemokines, and cellular adhesion molecules [11, 14]. Numerous studies have demonstrated the crucial role of IL-18 in neuroinflammation $[12,15,16]$. Previous studies have reported that IL-18 levels increase gradually over 3-7 days in rodents in astroglia and microglia [17-19]. Administration of IL-18-binding protein (IL-18BP), an IL-18 antagonist, has no effect on neurological recovery and brain edema at 1 day post injury, but it does ameliorate neurological deficits after 7 days, suggesting that IL-18 may contribute to pathogenesis during the later phases of TBI in rodents $[19,20]$. Clinical studies suggest that increased levels of serum IL-18 are associated with the exacerbation of long-term outcomes in TBI patients [21]. These studies suggest that IL-18 may contribute to the repair process after TBI in rodents, which is worth exploring.

In the current study, using a well-characterized controlled cortical impact (CCI) injury model, we assessed the roles of IL-18 in inflammatory and neurodegenerative pathology in genetically engineered II-18 knockout (II18-KO) mice. We found that the expression of IL-18 was increased, which was accompanied by robust reactive glial activation. Genetic deletion of II-18 resulted in a decreased neuroinflammatory response, reduced cell death, and ameliorated neurological deficits. These results show that IL-18 may be an important target in the regulation of TBI-induced neuroinflammation.

\section{Methods}

\section{Animals}

Male C57BL/6 (wild-type, WT) and I/18-KO (Stock No: 004130, purchased from the Jackson laboratory) mice (8-12 weeks; $20-26 \mathrm{~g}$ ) were used in this study [22]. The mice were housed at constant temperature and humidity under a 12-hour light/dark cycle. Food and water were provided ad libitum. I/18-KO mice were genotyped by PCR amplification of genomic DNA extracted from tail snips (Fig. S1a). The II-18 mRNA level in the mouse brain was also determined by qRT-PCR (Fig. S1b). The protocols for animal experiments were approved by the China Medical University Animal Care and Use Committee.

\section{Traumatic brain injury}

Moderate TBI was induced according to a CCI injury model that was described previously with slight modifications [23-25]. Briefly, mice were anesthetized, shaved and maintained with $2.5 \%$ isoflurane with a 
nonrebreathing nose cone and passive exhaust system connected to a stereotaxic frame. Body temperature was maintained at $37^{\circ} \mathrm{C}$ during the surgical procedure. The skull was exposed by making a midline incision, and a craniotomy approximately $4 \mathrm{~mm}$ in diameter was made using an electric microdrill on the central aspect of the left parietal bone, with the center of the opening located $2.5 \mathrm{~mm}$ posterior to the bregma and $2.5 \mathrm{~mm}$ lateral to the sagittal suture. The bone flap was removed without damage to the exposed underlying dura. Any animal with a disrupted dura was omitted from the study and replaced by another littermate. The contusion device consists of a computer-controlled electromagnetic driven impactor, with a 3-mm-diameter tip (Pinpoint PCl3000 Precision Cortical Impactor). The manipulator arm was rotated $20^{\circ}$ on the vertical axis, to allow impact flush with the dura. Moderate injury was delivered by using this device to compress the cortex to a depth of $1.0 \mathrm{~mm}$ at a velocity of $1.5 \mathrm{~m} / \mathrm{s}$ and duration of 50 $\mathrm{ms}$. Then, the incision was sutured closed, and the animal was allowed to recover on a heating pad. Sham animals underwent the same procedures (including craniotomy) as injured mice except for the impact protocol.

In this study, mice survived for 3 or 7 days post injury (dpi), depending on group assignment. A total of 48 $W T$ and $36 / / 18-\mathrm{KO}$ mice were assessed, and six mice were randomly included in each group. Investigators were blinded to the treatment groups during experimental assays and data analysis according to published guidelines.

\section{Processing of brain tissue for histology}

At 3 and $7 \mathrm{dpi}$, mice were deeply anesthetized with an overdose of inhaled isoflurane and then transcardially perfused with cold phosphate-buffered saline (PBS) followed by $4 \%$ paraformaldehyde (PFA) at $4^{\circ} \mathrm{C}$. Brains were dissected and post-fixed with $4 \%$ PFA for 24 hours at $4^{\circ} \mathrm{C}$. The tissues were then dehydrated with ethanol, cleared in xylene, and paraffin embedded. Serial coronal sections $(4 \mu \mathrm{m})$ were cut and mounted on glass slides, followed by immunofluorescence staining. Fluoro-Jade C (FJC) staining and terminal deoxynucleotidyl transferase UTP nick end labeling (TUNEL) staining were performed. Images were acquired with a Leica DMi8 fluorescence microscope (Leica, Germany). Mice harvested for qRT-PCR and Luminex were perfused with cold PBS only, and the ipsilateral cortex was collected.

\section{Immunofluorescence}

Paraffin-embedded sections were dewaxed, hydrated, and incubated in 5\% donkey serum in $1 \times$ PBS. Then, the sections were incubated overnight with primary antibodies at $4^{\circ} \mathrm{C}$ and then with secondary antibodies for 2 hours at room temperature. Nuclei were stained with DAPI. The primary and secondary antibodies used in our present studies were as follows: rabbit anti-IL18 (1:400, Abcam, ab71495), mouseanti-NeuN (1:400, Millipore, MAB377), rabbit anti-MAP2 (1:400, Proteintech, 17490-1-AP), mouse antiGFAP (1:400, Invitrogen, 14-9892-82), rabbit anti-IBA1 (1:1,000, Wako, 019-19741), mouse anti-IBA1 (1:400, Millipore, MABN92), goat anti-CD32 (1:100, R\&D Systems, AF1460), goat anti-CD206 (1:100, R\&D Systems, AF2535-SP), Alexa Fluor 488-conjugated donkey anti-rabbit (Invitrogen, A-21206), Alexa Fluor 594-conjugated donkey anti-rabbit (Invitrogen, A-21207), Alexa Fluor 488-conjugated donkey anti-mouse (Invitrogen, A-21202) and Alexa Fluor 594-conjugated donkey anti-mouse (Invitrogen, A-21203). Antigen 
retrieval was performed by incubating the sections in citrate buffer at $95^{\circ} \mathrm{C}$ for 5 minutes. The specificity of immunofluorescence reactions was evaluated by replacing the respective primary antibody with PBS. Images were acquired at the same magnification (400x) for coronal brain sections. In each animal, six sections at the same location were selected for comparison, and ten random fields without overlaps in each section were evaluated. Positive cells were counted by an investigator blinded to experimental grouping. The regions of interest are shown in Fig. 1a.

\section{Fluoro-Jade C (FJC) staining}

Fluoro-Jade C (FJC) is a polyanionic fluorescein derivative that specifically stains all degenerating neurons, regardless of the mechanism of cell death. FJC staining of brain sections was performed according to our published protocol [26]. First, sections were deparaffinized through two 10-minutes incubations with xylene and rehydrated in a graded alcohol series. The sections were then incubated in a $0.06 \%$ potassium permanganate solution for 10 minutes, followed by rinsing with distilled water for 2 minutes and incubation in a $0.0001 \%$ solution of FJC (Histo-Chem Inc.) dissolved in $0.1 \%$ acetic acid.

\section{TUNEL staining}

Apoptosis was assessed using a TdT-mediated dUTP nick end labeling (TUNEL) detection kit (Beyotime, C1086) according to our published protocol [26]. Briefly, sections were deparaffinized with xylene and dehydrated with a graded ethanol series. Then, the sections were digested with proteinase $\mathrm{K}$ (Beyotime, ST533) for 15 minutes. After several washes with PBS, the sections were further incubated with $40 \mu \mathrm{l}$ of the TUNEL reaction mixture containing terminal deoxynucleotidyl transferase (TdT) and FITC-labeled dUTP at $37^{\circ} \mathrm{C}$ for 2 hours in the dark. Nuclei were stained with DAPI. The apoptotic index (Al), which was defined as the average percentage of TUNEL-positive cells in ten cortical microscopic fields $(\times 400)$ in each section, was used to demonstrate the extent of brain damage. The final average of the percentage of TUNEL-positive cells in six sections per animal was considered.

\section{Gene expression assessment}

To isolate total RNA from the ipsilateral cortex, tissue samples were homogenized in TRIzol reagent (Invitrogen) according to the manufacturer's protocol. The isolated total RNA was precipitated out using isopropanol, washed with ethanol, and then dissolved in DEPC-treated water. Total RNA concentrations were determined using ultraviolet spectrophotometry, with 260/280 ratios of 1.8-2.2 considered acceptable. The isolated total RNA was then converted into cDNA using a PrimeScript ${ }^{\mathrm{TM}}$ RT reagent kit (Takara, RR047A) according to the manufacturer's instructions. TB Green ${ }^{\text {TM }}$ Premix Ex Taq ${ }^{\text {TM }}$ II (Takara, RR820A) was used to perform quantitative real-time PCR amplification according to the instructions provided by the manufacturer. Primer sequences are shown in Fig. S1c. Reactions were amplified and quantified by using a Light Cycler 480 real-time qPCR system and the corresponding software (Roche).

The PCR profile consisted of 1 cycle at $95^{\circ} \mathrm{C}$ for $30 \mathrm{~s}$, followed by 40 cycles at $95^{\circ} \mathrm{C}$ for $5 \mathrm{~s}$ and $60{ }^{\circ} \mathrm{C}$ for 20 s. Gene expression was normalized to Gapdh expression, and the relative quantity of mRNAs was 
analyzed by the $\Delta \Delta \mathrm{CT}$ method [27]. All PCR reactions were performed in triplicate.

\section{Neurological severity score (NSS)}

A 10-point NSS was used to quantify neurological deficits after TBI as described previously [28]. The score consists of 10 individual tasks, including exit circle, monoparesis/hemiparesis, straight walk, startle reflex, seeking behavior, beam balancing, round stick balancing, beam walk $(3 \mathrm{~cm})$, beam walk $(2 \mathrm{~cm})$ and beam walk $(1 \mathrm{~cm})$. One point is given for failing to perform each of the tasks. A maximal NSS of 10 points thus indicates severe neurological deficits, with failure of all tasks.

\section{Multiplex assessment of cytokines and chemokines}

Tissue samples from the cortical area surrounding the ipsilateral injury site were collected and homogenized with a cytokine extraction kit (Absin, abs9225). The homogenates were centrifuged at $14,000 \mathrm{~g}$ for 15 minutes at $4^{\circ} \mathrm{C}$ to collect the tissue lysate. Protein concentrations were quantified with a BCA assay kit (Beyotime, P0009). The samples were analyzed using a Luminex X-200 instrument and two premade kits for 17 cytokines (including IL-1a, IL-1ß, IL-2, IL-3, IL-4, IL-5, IL-6, IL-9, IL-10, IL-12p40, IL12p70, IL-13, IL-17A, G-CSF, GM-CSF, IFN-y, and TNF-a) and 18 chemokines (including CCL2/MCP-1, CCL3/MIP-1a, CCL4/MIP-13, CCL5/RANTES, CCL7, CCL8, CCL11/Eotaxin-1, CCL12, CCL19, CCL20, CCL21, CCL22, CXCL1/GRO-a/KC, CXCL2/GRO- $\beta$, CXCL10, CXCL12, CXCL13, and CXCL16). Cytokines and chemokines were analyzed using Luminex technology following the manufacturer's specifications (Bio-Plex Pro Mouse Cytokine 23-plex, Bio-Rad, M60009RDPD; Mouse Magnetic Luminex Assay (17 Plex), R\&D Systems, LXSAMSM-17).

\section{Statistical analysis}

All statistical analyses were performed with GraphPad Prism 7 software. All data are expressed as the mean \pm standard deviation (SD). Two-way ANOVA was performed for comparisons among groups. Tukey's post hoc analysis was used to determine where differences occurred. $p<0.05$ was considered statistically significant.

\section{Results}

\section{IL-18 expression is upregulated in the injured ipsilateral cortex after TBI}

Studies have previously reported that IL-18 levels increase significantly at 3-7 dpi [17-19], suggesting the involvement of IL-18 in the pathogenic processes of TBI. To assess the expression level of IL-18 in the injured ipsilateral cortex in our study, we determined $I /-18$ gene expression using qRT-PCR and performed immunofluorescence staining using an antibody specific to IL-18. II-18 mRNA levels in the injured ipsilateral cortex were increased significantly in the TBI groups compared with the sham groups at 3 and $7 \mathrm{dpi}$ (Fig. 1b). Immunofluorescence staining revealed that IL-18 expression was increased in the injured ipsilateral cortex at 3 and 7 dpi (Fig. 1c). To identify the cell types that expressed IL-18 post TBI, we performed double immunostaining with an anti-IL-18 and a neuron-specific anti-NeuN antibody, astrocyte- 
specific anti-GFAP antibody, or microglia-specific anti-IBA1 antibody. Double immunofluorescence indicated that IL-18 was mainly colocalized with IBA1+ microglia and NeuN+ neurons, but not with GFAP+ astrocytes, at 7 dpi (Fig. 1d).

\section{II-18 deficiency ameliorates neurological deficits caused by TBI}

To assess the role of IL-18 in neurological deficits, a 10-point NSS was assessed. As shown in Fig. 2, NSS scores were significantly increased in TBI mice compared with sham animals at 3 and $7 \mathrm{dpi}$ in $W T$ and I/18-KO mice (Fig. 2). However, deficiency in II-18 resulted in significant decreases of NSS at 3 and 7 dpi compared with that in $W T$ mice (Fig. 2).

\section{Deletion of II-18 attenuates neuronal degeneration and cell death after TBI}

To explore the role of IL-18 in neuron survival after TBI, immunostaining for MAP2, a neuron-specific biomarker, was carried out. As shown in Fig. 3, the number of MAP2+ cells was decreased at 3 and $7 \mathrm{dpi}$ in $W T$ and $/ 118-K O$ mice compared with sham mice (Fig. 3). More neurons survived in the mice with II-18 deletion than in the $W T$ mice (Fig. 3).

TBI is associated with severe neurodegeneration. We performed FJC staining to examine the degenerated cells in the ipsilateral cortex after TBI. Increased numbers of FJC+ cells were detected in the cortex at 3 and $7 \mathrm{dpi}$ in $W T$ and I/18-KO mice compared with sham animals (Fig. 4). Deficiency in I-18 prevented TBIinduced neuronal degeneration, with evidence demonstrating that markedly fewer FJC-positive cells were found post-injury in I/18-KO mice than in WT mice (Fig. 4).

To evaluate apoptotic cell death in the pericontusional area of the ipsilateral cortex, an in situ TUNEL assay was conducted. As shown in Fig. 5, TUNEL+ cells were evident at 3 and $7 \mathrm{dpi}$ in the pericontusional tissue. The apoptotic index (AI) increased significantly in the TBI groups at 3 and $7 \mathrm{dpi}$ in $W T$ and $/ / 18$-KO mice compared with that in the sham groups (Fig. 5). Quantitative analysis indicated that mice with II-18 deletion showed lower Al post trauma than WT animals (Fig. 5b).

\section{TBI-induced astrogliosis is attenuated in //18KO mice}

Astrocytes and microglia are considered key players in initiating an inflammatory response after injury. To explore the effect of IL-18 on the TBI-induced inflammatory response, we first evaluated astrogliosis in the ipsilateral cortex by GFAP immunostaining at 3 and $7 \mathrm{dpi}$. After TBI, GFAP+ cells were activated and increased significantly, showing a hypertrophic morphology involving swelling of cell bodies and extension of processes (Fig. 6). The number and percent area of GFAP+ cells increased significantly at 3 and $7 \mathrm{dpi}$ in $W T$ and $/ / 18-\mathrm{KO}$ mice (Fig. 6b, c). Mice with II-18 deletion exhibited fewer GFAP+ cells and a lower percent area of GFAP+ cells in the injured ipsilateral cortex compared with WT animals at 3 and 7 dpi (Fig. 6b, c). Furthermore, GFAP+ reactive astroglia in WT mice showed bulky and long branches with very few secondary processes, while GFAP+ astroglia emanated slender and relatively short primary processes with numerous secondary processes in mice with II-18 deletion (Fig. 6a lower panel), suggesting that IL-18 may participate in the activation of astrocytes. 


\section{Lack of II-18 attenuates TBI-induced microgliosis and the polarization of microglia into the M1-like 'proinflammatory' subtype}

Next, due to the contribution of microglia in TBI, we detected activated microglia by immunostaining for the microglia-specific marker IBA1. In the pericontusional tissue of sham mice, a few quiescent microglia were found with small cell bodies and a fine appearance. Microglia/macrophages were rapidly increased, as observed by IBA 1 immunostaining, and exhibited a deramified and hypertrophic morphology after TBI (Fig. 7). The number and percent area of IBA1+ cells increased significantly at 3 and 7 dpi in WT and I/18$\mathrm{KO}$ mice (Fig. 7b, c). Mice with II-18 deletion exhibited fewer IBA1+ cells and a lower percent area of IBA1+ cells in the injured ipsilateral cortex than $W T$ animals at 7 dpi (Fig. 7b, c).

Microglial polarization has been shown to vary over time and between different TBI models. M1-like 'proinflammatory' and M2-like 'anti-inflammatory' microglia/macrophages are characterized by different gene expression patterns and have differential functions in injury $[7,9]$. To explore the effect of IL-18 on M1/M2 polarization following TBI, we evaluated the M1 phenotype by CD32 immunostaining and the M2 phenotype by CD206 immunostaining at $7 \mathrm{dpi}$ in the ipsilateral cortex. IBA1 was used as a microgliaspecific marker. At $7 \mathrm{dpi}$, CD32+ microglia accounted for $69.38 \%$ of IBA1+ microglia, while CD206+ microglia accounted for $29.37 \%$ of IBA $1+$ microglia in WT mice (Fig. 8). Mice with II-18 deletion exhibited a lower percentage of CD32+ microglia (38.4\%) and a higher percentage of CD206+ microglia (55.06\%) in the injured ipsilateral cortex than WT animals at 7 dpi (Fig. 8).

\section{II-18 deficiency attenuates the TBI-induced secretion of inflammatory cytokines and chemokines}

A number of inflammatory cytokines and chemokines have been recognized to participate in the response to TBI. To explore the role of IL-18 in the TBI-induced secretion of inflammatory cytokines and chemokines, we evaluated cytokine and chemokine expression in the ipsilateral cortex at $3 \mathrm{dpi}$ using a Luminex assay. The levels of cytokines, including IL-1a, IL-1 1 , IL-2, IL-3, IL-4, IL-5, IL-6, IL-9, IL-10, IL-12p40, IL-12p70, IL-13, IL17A, G-CSF, GM-CSF, IFN- $y$, and TNF-a, were upregulated significantly in the injured ipsilateral cortex (Fig. 9, Fig. S2 and Table S1). Mice with II-18 deletion exhibited decreases in the secretion of all of the above cytokines (Fig. 9, Fig. S2 and Table S1). The levels of chemokines, including CCL2/MCP-1, CCL3/MIP-1a, CCL4/MIP-1 $\beta$, CCL5/RANTES, CCL7, CCL8, CCL11/Eotaxin-1, CCL12, CCL20, CCL21, CCL22, CXCL1/GRO- $\alpha / K C, C X C L 2 / G R O-\beta, C X C L 10, C X C L 12, C X C L 13$ and CXCL16, were upregulated significantly in the injured ipsilateral cortex (Fig. 10, Fig. S3 and Table S2). Deletion of II-18 resulted in significant decreases in the levels of numerous chemokines including CCL2/MCP-1, CCL3/MIP-1a, CCL4/MIP-1 $\beta$, CCL5/RANTES, CCL7, CCL12, CCL20, CXCL1/GRO-a, CXCL2/GRO- $\beta$, CXCL10, CXCL12, CXCL13 and CXCL16 (Fig. 10, Fig. S3 and Table S2), while the levels of some chemokines (including CCL8, CCL11/Eotaxin-1, CCL19, CCL21, and CCL22) were not affected (Fig. 10, Fig. S3 and Table S2).

\section{Discussion}


Increasing evidence suggests that IL-18 plays a pivotal role in brain injury $[19,29,30]$. However, the underlying mechanism remains unclear. The current study has demonstrated that IL-18 levels are increased and that II-18 deficiency is neuroprotective in TBI. We observed TBI-induced expression of IL-18 in microglia and neurons post TBI. II-18 deficiency decreased TBI-induced neuronal degeneration and apoptosis, increased neuron survival, and ultimately resulted in amelioration of neurological deficits. This protective effect is likely mediated through amelioration of neuroinflammation by attenuation of TBIinduced astrogliosis and microglial activation and reductions in inflammatory cytokine and chemokine expression. We also observed that II-18 deficiency reduced the frequency of TBI-induced M1-like 'proinflammatory' microglia. The present results provide substantial evidence that IL-18 is involved in the development of secondary injury post TBI and that IL-18 is an important regulator in the process of TBIinduced inflammation.

IL-18 is a proinflammatory cytokine and an important mediator of peripheral inflammation and the host immune response. IL-18 can have autocrine and paracrine effects by binding with the IL-18R, which consists of two chains, an IL-18-binding alpha chain (IL-18Ra) and a signaling beta chain (IL-18Rß) [31]; this interaction leads to activation of NF-KB signaling via a complex intracellular signaling cascade, culminating in the production and release of several cytokines, chemokines, and cellular adhesion molecules $[12,14,32]$. Several studies have demonstrated that IL-18 has important functions in the central nervous system (CNS), which initiates the inflammatory signaling cascade that contributes to neuronal injury and cell death $[16,20,33]$. Increased levels of IL-18 were observed at 3 and $7 \mathrm{dpi}$, which is consistent with previous studies [17-19]. Previous studies have shown an increase in IL-18 expression in microglia post hypoxic-ischemic brain injury, kainic acid (KA)-induced excitotoxicity), or spinal nerve ligation (SNL) [30, 34, 35]; in neurons following restraint stress [36]; and in astrocytes in vitro [37]. In the present study, we observed expression of IL-18 mainly in microglia and neurons, not in astrocytes at $7 \mathrm{dpi}$ in mice. The difference in cell type-specific expression of IL-18 may result from the use of different models, observation areas or animal species. Recent studies have found that the release of IL-1 $\beta$ and IL18 is mediated by NLRP3 inflammasomes, which are important in the inflammatory process [38]. High levels of IL-18 are considered to be crucial for the establishment of a chronically inflamed environment, leading to neuronal dysfunction and eventually neurodegeneration, while IL-1 $\beta$ is involved in the early phase of TBI [20,39]. IL-18 is less studied post TBI. To date, researchers have demonstrated increased levels of IL-18 post TBI in mice, which result in the exacerbation of neurological deficits and apoptosis [17-19]. In the present study, II-18 deficiency resulted in amelioration of neurological deficits, which was associated with decrease in TBI-induced neuronal degeneration and neuroinflammation, suggesting the adverse effects of IL-18 on brain injury.

Neuroinflammation is a major pathological process in the secondary response after TBI [26, 40, 41]. Post $\mathrm{TBI}$, the release of the intracellular components of damaged cells activates neuroinflammatory responses, involving activation of microglia and astrocytes and production of cytokines and chemokines, which significantly contribute to substantial neural damage and behavior impairment $[5,26]$. Researchers have shown that administration of an IL-18 antagonist (IL-18BP or other IL-18 neutralizing antibody) suppresses NF-KB activation and decreases the levels of inflammatory mediators including TNF-a, IL-1 $\beta$, 
IL-4, IL-6, IL-10, and CXC chemokines following reperfusion in mouse models of ischemia/reperfusion injury [42-45]. In addition, decreased expression levels of inflammatory markers, such as TNF- $a$, IL-6, IL10, IL-12p40, and CXCL1, are found in II-18 deficient mice with pneumococcal meningitis [46]. Here, we demonstrated that the TBI-induced secretion of pro- and anti-inflammatory cytokines, including IL-1a, IL13, IL-2, IL-3, IL-4, IL-5, IL-6, IL-9, IL-10, IL-12p40, IL-12p70, IL-13, IL17A, G-CSF, GM-CSF, IFN- $y$, and TNF-a, and chemokines, including CCL2, CCL3, CCL4, CCL5, CCL7, CCL12, CCL20, CXCL1, CXCL2, CXCL10, CXCL12, CXCL13 and CXCL16, was downregulated in II18-KO mice, suggesting that IL-18 may participate in the regulation of cytokines and chemokines post TBI. To our knowledge, we are the first to assess the levels of TBI-induced cytokines and chemokines systematically in II18-KO mice.

Astrocytes and microglia, which can secret and response to various cytokines and chemokines, are crucial in inflammatory cascades post TBI [7]. After TBI, astrocytes are activated, showing a hypertrophic morphology involving swelling of cell bodies and extension of processes, and the number of astrocytes increases significantly and peaks at $7 \mathrm{dpi}$ [41]. Recent investigations have shown that astrocytes can produce numerous inflammatory mediators, including IL-1 $1 \beta$, IL-6, IL-10, IL-17, TNF-a, CCL2, CCL5, CCL7, CCL8, CCL12, CXCL1, CXCL10, CXCL12 and CXCL16 [47]. Multiple reports provide evidence that IL-18 is involved in neuroinflammation in a variety of experimental models, including brain injury $[12,15,16]$. Previous studies have shown that treatment with an anti-IL-18 antibody blocks the activation of astrocytes in an SNL model [35], while II18-deficient mice exhibit enhanced KA-induced astrogliosis [48]. In the present study, II-18 deficiency attenuated TBI-induced astrogliosis, with astrocytes showing relatively small cell bodies with slender, relatively short and branched processes, which may contribute to the reductions in inflammatory cytokine and chemokine levels observed post TBI in II18-KO mice in our study.

Microglia are considered a key player in initiating inflammatory responses post injury [7]. During neuroinflammation, IL-18 may have an important role in the activation of microglia. IL-18-mediated microglial activation potentiates caspase-1 expression, metalloproteinase expression, and proinflammatory cytokine production, which augment inflammatory responses in the brain [30,49]. After $\mathrm{TBI}$, microglia become activated, and the cells number peaks at $7 \mathrm{dpi}$ [41]. Microglia release proinflammatory cytokines, including IL-1 $\beta$, IL-6, IL-12, and TNF-a, and chemokines, including CCL2, CCL3, CCL5, CXCL10, and CXCL12 [50]. Activated microglia are described by the nomenclature 'M1' and 'M2' [7, 9]. Microglia polarize from a resting phenotype into the M1-like phenotype after exposure to dangerassociated molecular patterns (DAMPs), free radicals, or proinflammatory cytokines such as IFN- $\gamma$, TNF-a and IL-6 [9]. M1-like cells are characterized by the production of proinflammatory cytokines (e.g. IL-1 $\beta$, IL6, IL-12p40, IL-18, and TNF-a) and chemokines (e.g. CCL2, CCL3, CCL4, and CXCL9), reactive oxygen species (ROS) generation, and reduced phagocytic activity [7, 9, 50, 51]. M1-like 'pro-inflammatory' cells are considered neurotoxic following brain injury $[7,52]$. In response to anti-inflammatory cytokines (e.g. IL-4 adn IL-13), microglia polarize towards the M2-like phenotype, which is considered neuroprotective [7, 9, 52]. M2-like microglia are associated with the production of anti-inflammatory cytokines (e.g. IL4, IL-10, and IL-13), chemokines (e.g. CCL22) and trophic factors, and increased phagocytic activity [50, 53, 54]. Microglia can synthesize and respond to IL-18 [55]. Previous studies have shown that II-18 deficiency 
reduces stress and KA-induced microglial activation, possibly by reducing IFN-y expression [56, 57]. Furthermore, recent studies show a reduction in the IL-18 level may be related to inhibition of the activation of M1 microglia [58] and may induce microglial polarization towards the M2 profile [59]. Previous studies have shown that suppressing M1 phenotype activation and promoting M2 phenotype activation of microglia can rescue damaged neurons, reduce lesion volume, and improve neurological functional recovery following TBI [60-62]. In this study, although the levels of the cytokines IL-4 and IL13 , which can induce microglial polarization towards the M2-like phenotype, were downregulated, M2-like polarization was still upregulated post TBI in II18-KO mice, suggesting that TBI-induced M2-like polarization in II18-KO mice may be induced by other more important mechanisms [9]. Furthermore, II-18 deficiency reduced the frequency of TBI-induced M1-like 'proinflammatory' microglia, which may be the result of II-18 deficiency-induced decrease in the levels of IFN- $y$, TNF-a and IL6, which can induce microglial polarization towards the M1-like phenotype. In conclusion, IL-18 promotes microglial activation and polarization towards the M1-like phenotype post TBI, which enhances microglial caspase- 1 and IL-18 expression and forms a feed-forward loop to subsequently promote the inflammatory response [12].

Although we found an important role for IL-18 in glial activation and numerous cytokines and chemokines, there are still some topics that need to be addressed in future work. First, in the present study, we used only global II18-KO mice to illustrate the role of IL-18 in TBI-induced neuroinflammation. Thus, the effects of II-18 ablation on other organs or tissues in the pathogenesis of secondary injury after TBI cannot be neglected. It would be better to illustrate the role of IL-18 with glia-specific conditional knockout mice. Furthermore, we explored the role of IL-18 in neuroinflammation only through assessing glial activation and the expression of cytokines and chemokines post TBI. Further studies are needed to explore the relationship between IL-18 and the NLRP3 inflammasome, because NLRP3-mediated inflammation is regarded as a key pathway in the process of pyroptosis $[20,38]$. We are conducting an experiment to evaluate the effects of IL-18 on pyroptosis in the late phase of TBI. Finally, more animals in each group are needed to better control for variation.

\section{Conclusion}

Current research demonstrates that genetic deletion of II-18 results in decreased neuroinflammatory responses (including glial activation and effects on the secretion of cytokines and chemokines), cell death (degeneration and apoptosis), and neurological deficits, suggesting that the effect of IL-18 on TBI is associated with the regulation of neuroinflammation, which highlights the role of IL-18 in secondary brain injury and suggests that targeting IL-18 could have therapeutic potential in the treatment of TBI.

\section{Abbreviations}

TBI: traumatic brain injury; CCl: controlled cortical impact; IL: Interleukin; dpi: days post injury; WT: wild type; I/18-KO: II18-knockout; IFN-ץ: interferon-ү; IL-18R: IL-18 receptor; IL-18BP: IL-18-binding protein; PBS: phosphate-buffered saline; PFA: paraformaldehyde; FJC: Fluoro-Jade C; TUNEL: terminal deoxynucleotidyl transferase UTP nick end labeling; NSS: neurological severity score; G-CSF: Granulocyte-colony 
stimulating factor; GM-CSF: granulocyte-macrophage colony-stimulating factor; DAMPs: dangerassociated molecular pattern; $\mathrm{CCL}$ : chemokine (C-C motif) ligand; $\mathrm{CXCL}$ : chemokine (C-X-C motif) ligand; CNS: central nervous system.

\section{Declarations}

\section{Acknowledgements}

Not applicable.

\section{Funding}

This work was partly supported by grants from the National Natural Science Foundation of China $(81971793,81772023,81671862,81871529,81801874 \llbracket 81660309)$, the Liaoning Natural science Foundation of China (2019-MS-392) and the Youth Backbone Supporting Funding of China Medical University (QGZ2018089).

\section{Availability of data and materials}

All data generated and/or analyzed during the current study are available from the corresponding author on reasonable request.

\section{Authors' Contributions}

All authors performed the experiments. WD analyzed the results and wrote the manuscript. RZ designed the study and edited the manuscript. All authors read and approved the final manuscript.

\section{Ethics approval and consent to participate}

The protocols for animal experiments were approved by the China Medical University Animal Care and Use Committee.

\section{Consent for publication}

Not applicable.

\section{Competing interests}

The authors declare that they have no competing interests.

\section{Publisher's Note}

Springer Nature remains neutral with regard to jurisdictional claims in published maps and institutional affiliations.

\section{Author details}


${ }^{1}$ Department of Forensic Pathology, China Medical University School of Forensic Medicine, Shenyang 110122, China.

${ }^{2}$ Department of Histology and Embryology, College of Basic Medical Sciences, China Medical University, Shenyang 110122, China.

${ }^{3}$ Collaborative laboratory of Intelligentized Forensic Science, Shenyang 110034, China.

${ }^{4}$ The first affiliated hospital of China medical university, Shenyang,110001, China.

${ }^{5}$ School of Forensic Medicine, Guizhou Medical University, Guiyang 550004, China.

\section{Supplemental Information Note}

\section{Additional file 1. Figure S1}
A. Genotype identification.
B. Gene expression levels of $I-18$ in $W T$ and $/ / 18-K O$ mice determined by qRT-PCR.
C. Primers used in the present study.

\section{Additional file 2. Figure S2}

II-18 deficiency attenuated the TBI-induced secretion of cytokines

The levels of cytokines in the injured ipsilateral cortex at $3 \mathrm{dpi}$ were evaluated by a Luminex assay. Data are presented as the mean $\pm S D ;{ }^{*} p<0.05$ vs. the sham group, $\# p<0.05$ vs. $W T$ mice $(n=6)$.

\section{Additional file 3. Figure S3}

II-18 deficiency attenuated the TBI-induced secretion of chemokines

The levels of chemokines in the injured ipsilateral cortex at 3 dpi were evaluated by a Luminex assay. Data are presented as the mean $\pm S D ;{ }^{*} p<0.05$ vs. the sham group, $\# p<0.05$ vs. WT mice $(\mathrm{n}=6)$.

\section{Additional file 4. Table S1}

Analysis of cytokine expression in the pericontusional cortex at 3 days post TBI. Data are presented as the mean $\pm S D ;{ }^{*} p<0.05$ vs. the sham group, $\# p<0.05$ vs. $W T$ mice $(n=6)$.

\section{Additional file 5. Table S2}

Analysis of chemokine expression in the pericontusional cortex at 3 days post TBI. Data are presented as the mean $\pm S D ;{ }^{*} p<0.05$ vs. the sham group, $\# p<0.05$ vs. $W T$ mice $(n=6)$. 


\section{References}

1. Jiang JY, Gao GY, Feng JF, Mao Q, Chen LG, Yang XF, Liu JF, Wang YH, Qiu BH, Huang XJ: Traumatic brain injury in China. Lancet Neuro/ 2019, 18:286-295.

2. Sun $\mathrm{H}$, Luo $\mathrm{C}$, Chen $\mathrm{X}$, Tao L: Assessment of cognitive dysfunction in traumatic brain injury patients: a review. Forensic Sci Res 2017, 2:174-179.

3. Maas A, Menon DK, Adelson PD, Andelic N, Bell MJ, Belli A, Bragge P, Brazinova A, Buki A, Chesnut $\mathrm{RM}$, et al: Traumatic brain injury: integrated approaches to improve prevention, clinical care, and research. Lancet Neurol 2017, 16:987-1048.

4. Xiong Y, Mahmood A, Chopp M: Animal models of traumatic brain injury. Nat Rev Neurosci 2013, 14:128-142.

5. Gyoneva S, Ransohoff RM: Inflammatory reaction after traumatic brain injury: therapeutic potential of targeting cell-cell communication by chemokines. Trends Pharmacol Sci 2015, 36:471-480.

6. Jassam YN, Izzy S, Whalen M, McGavern DB, El KJ: Neuroimmunology of Traumatic Brain Injury: Time for a Paradigm Shift. Neuron 2017, 95:1246-1265.

7. Karve IP, Taylor JM, Crack PJ: The contribution of astrocytes and microglia to traumatic brain injury. Br J Pharmacol 2016, 173:692-702.

8. Burda JE, Bernstein AM, Sofroniew MV: Astrocyte roles in traumatic brain injury. Exp Neuro/ 2016, 275 Pt 3:305-315.

9. Simon DW, McGeachy MJ, Bayir H, Clark RS, Loane DJ, Kochanek PM: The far-reaching scope of neuroinflammation after traumatic brain injury. Nat Rev Neurol 2017, 13:171-191.

10. Colton CA: Heterogeneity of microglial activation in the innate immune response in the brain. $J$ Neuroimmune Pharmacol 2009, 4:399-418.

11. Yasuda K, Nakanishi K, Tsutsui H: Interleukin-18 in Health and Disease. Int J Mol Sci 2019, 20.

12. Felderhoff-Mueser U, Schmidt OI, Oberholzer A, Buhrer C, Stahel PF: IL-18: a key player in neuroinflammation and neurodegeneration? Trends Neurosci 2005, 28:487-493.

13. Gracie JA, Robertson SE, McInnes IB: Interleukin-18. J Leukoc Biol 2003, 73:213-224.

14. Rex D, Agarwal N, Prasad T, Kandasamy RK, Subbannayya Y, Pinto SM: A comprehensive pathway map of IL-18-mediated signalling. J Cell Commun Signal 2019.

15. Novick D, Kim S, Kaplanski G, Dinarello CA: Interleukin-18, more than a Th1 cytokine. Semin Immunol 2013, 25:439-448.

16. Alboni S, Cervia D, Sugama S, Conti B: Interleukin 18 in the CNS. J Neuroinflammation 2010, 7:9.

17. Liu HD, Li W, Chen ZR, Hu YC, Zhang DD, Shen W, Zhou ML, Zhu L, Hang CH: Expression of the NLRP3 inflammasome in cerebral cortex after traumatic brain injury in a rat model. Neurochem Res 2013, 38:2072-2083.

18. Sifringer M, Stefovska V, Endesfelder S, Stahel PF, Genz K, Dzietko M, Ikonomidou C, FelderhoffMueser U: Activation of caspase-1 dependent interleukins in developmental brain trauma. Neurobiol 
Dis 2007, 25:614-622.

19. Yatsiv I, Morganti-Kossmann MC, Perez D, Dinarello CA, Novick D, Rubinstein M, Otto VI, Rancan M, Kossmann T, Redaelli CA, et al: Elevated intracranial IL-18 in humans and mice after traumatic brain injury and evidence of neuroprotective effects of IL-18-binding protein after experimental closed head injury. J Cereb Blood Flow Metab 2002, 22:971-978.

20. Voet S, Srinivasan S, Lamkanfi M, van Loo G: Inflammasomes in neuroinflammatory and neurodegenerative diseases. Embo Mol Med 2019, 11.

21. Ciaramella A, Della VC, Salani F, Viganotti M, D'Ippolito M, Caltagirone C, Formisano R, Sabatini U, Bossu P: Increased levels of serum IL-18 are associated with the long-term outcome of severe traumatic brain injury. Neuroimmunomodulat 2014, 21:8-12.

22. Takeda K, Tsutsui H, Yoshimoto T, Adachi O, Yoshida N, Kishimoto T, Okamura H, Nakanishi K, Akira S: Defective NK cell activity and Th1 response in IL-18-deficient mice. Immunity 1998, 8:383-390.

23. Ma X, Aravind A, Pfister BJ, Chandra N, Haorah J: Animal Models of Traumatic Brain Injury and Assessment of Injury Severity. Mol Neurobiol 2019, 56:5332-5345.

24. Osier ND, Dixon CE: The Controlled Cortical Impact Model: Applications, Considerations for Researchers, and Future Directions. Front Neurol 2016, 7:134.

25. Smith DH, Soares HD, Pierce JS, Perlman KG, Saatman KE, Meaney DF, Dixon CE, McIntosh TK: A model of parasagittal controlled cortical impact in the mouse: cognitive and histopathologic effects. J Neurotrauma 1995, 12:169-178.

26. Dong W, Yang B, Wang L, Li B, Guo X, Zhang M, Jiang Z, Fu J, Pi J, Guan D, Zhao R: Curcumin plays neuroprotective roles against traumatic brain injury partly via Nrf2 signaling. Toxicol Appl Pharmacol 2018, 346:28-36.

27. Livak KJ, Schmittgen TD: Analysis of relative gene expression data using real-time quantitative PCR and the 2(-Delta Delta C(T)) Method. Methods 2001, 25:402-408.

28. Flierl MA, Stahel PF, Beauchamp KM, Morgan SJ, Smith WR, Shohami E: Mouse closed head injury model induced by a weight-drop device. Nat Protoc 2009, 4:1328-1337.

29. McKee CA, Lukens JR: Emerging Roles for the Immune System in Traumatic Brain Injury. Front Immunol 2016, 7:556.

30. Hedtjarn M, Leverin AL, Eriksson K, Blomgren K, Mallard C, Hagberg H: Interleukin-18 involvement in hypoxic-ischemic brain injury. J Neurosci 2002, 22:5910-5919.

31. Dinarello CA, Novick D, Kim S, Kaplanski G: Interleukin-18 and IL-18 binding protein. Front Immunol 2013, 4:289.

32. Sugama S, Conti B: Interleukin-18 and stress. Brain Res Rev 2008, 58:85-95.

33. Allan SM, Tyrrell PJ, Rothwell NJ: Interleukin-1 and neuronal injury. Nat Rev Immuno/ 2005, 5:629640.

34. Jeon GS, Park SK, Park SW, Kim DW, Chung CK, Cho SS: Glial expression of interleukin-18 and its receptor after excitotoxic damage in the mouse hippocampus. Neurochem Res 2008, 33:179-184. 
35. Miyoshi K, Obata K, Kondo T, Okamura H, Noguchi K: Interleukin-18-mediated microglia/astrocyte interaction in the spinal cord enhances neuropathic pain processing after nerve injury. $J$ Neurosci 2008, 28:12775-12787.

36. Sugama S, Cho BP, Baker H, Joh TH, Lucero J, Conti B: Neurons of the superior nucleus of the medial habenula and ependymal cells express IL-18 in rat CNS. Brain Res 2002, 958:1-9.

37. Conti B, Park LC, Calingasan NY, Kim Y, Kim H, Bae Y, Gibson GE, Joh TH: Cultures of astrocytes and microglia express interleukin 18. Brain Res Mol Brain Res 1999, 67:46-52.

38. Swanson KV, Deng M, Ting JP: The NLRP3 inflammasome: molecular activation and regulation to therapeutics. Nat Rev Immunol 2019, 19:477-489.

39. Bortolotti P, Faure E, Kipnis E: Inflammasomes in Tissue Damages and Immune Disorders After Trauma. Front Immunol 2018, 9:1900.

40. Cederberg D, Siesjo P: What has inflammation to do with traumatic brain injury? Childs Nerv Syst 2010, 26:221-226.

41. Dong W, Sun Y, Cheng H, Yang B, Wang L, Jiang Z, Li B, Wen S, Guo X, Guan D, Zhao R: Dynamic cell type-specific expression of Nrf2 after traumatic brain injury in mice. Eur J Neurosci 2019, 50:19811993.

42. Wang M, Tan J, Wang Y, Meldrum KK, Dinarello CA, Meldrum DR: IL-18 binding protein-expressing mesenchymal stem cells improve myocardial protection after ischemia or infarction. Proc Natl Acad Sci U S A 2009, 106:17499-17504.

43. Venkatachalam K, Prabhu SD, Reddy VS, Boylston WH, Valente AJ, Chandrasekar B: Neutralization of interleukin-18 ameliorates ischemia/reperfusion-induced myocardial injury. J Biol Chem 2009, 284:7853-7865.

44. Wang J, Long Q, Zhang W, Chen N: Protective effects of exogenous interleukin 18-binding protein in a rat model of acute renal ischemia-reperfusion injury. Shock 2012, 37:333-340.

45. Yang YJ, Chen SH, Ge XR: Role of interleukin-18 in the development of acute pulmonary injury induced by intestinal ischemia/reperfusion and its possible mechanism. $J$ Gastroenterol Hepatol 2007, 22:253-260.

46. Zwijnenburg PJ, van der Poll T, Florquin S, Akira S, Takeda K, Roord JJ, van Furth AM: Interleukin-18 gene-deficient mice show enhanced defense and reduced inflammation during pneumococcal meningitis. J Neuroimmunol 2003, 138:31-37.

47. Sofroniew MV: Astrocyte barriers to neurotoxic inflammation. Nat Rev Neurosci 2015, 16:249-263.

48. Zhang XM, Duan RS, Chen Z, Quezada HC, Mix E, Winblad B, Zhu J: IL-18 deficiency aggravates kainic acid-induced hippocampal neurodegeneration in C57BL/6 mice due to an overcompensation by IL-12. Exp Neuro/ 2007, 205:64-73.

49. Haque ME, Akther M, Jakaria M, Kim IS, Azam S, Choi DK: Targeting the microglial NLRP3 inflammasome and its role in Parkinson's disease. Mov Disord 2020, 35:20-33. 
50. Younger D, Murugan M, Rama RK, Wu LJ, Chandra N: Microglia Receptors in Animal Models of Traumatic Brain Injury. Mol Neurobio/ 2019, 56:5202-5228.

51. Sharma M, Arbabzada N, Flood PM: Mechanism underlying beta2-AR agonist-mediated phenotypic conversion of LPS-activated microglial cells. J Neuroimmunol 2019, 332:37-48.

52. Kigerl KA, Gensel JC, Ankeny DP, Alexander JK, Donnelly DJ, Popovich PG: Identification of two distinct macrophage subsets with divergent effects causing either neurotoxicity or regeneration in the injured mouse spinal cord. J Neurosci 2009, 29:13435-13444.

53. Gordon S: Alternative activation of macrophages. Nat Rev Immuno/ 2003, 3:23-35.

54. Sica A, Mantovani A: Macrophage plasticity and polarization: in vivo veritas. J Clin Invest 2012, 122:787-795.

55. Prinz M, Hanisch UK: Murine microglial cells produce and respond to interleukin-18. J Neurochem 1999, 72:2215-2218.

56. Zhang XM, Jin T, Quezada HC, Mix E, Winblad B, Zhu J: Kainic acid-induced microglial activation is attenuated in aged interleukin-18 deficient mice. J Neuroinflammation 2010, 7:26.

57. Sugama S, Fujita M, Hashimoto M, Conti B: Stress induced morphological microglial activation in the rodent brain: involvement of interleukin-18. Neuroscience 2007, 146:1388-1399.

58. Dai W, Wang X, Teng H, Li C, Wang B, Wang J: Celastrol inhibits microglial pyroptosis and attenuates inflammatory reaction in acute spinal cord injury rats. Int Immunopharmacol 2019, 66:215-223.

59. Bispo DSA, Cerqueira CP, Alves OAJ, Alves DACM, Pereira BJ, Dos SSC, Dias CM, Mecha M, Guaza RC, Amaral DSV, Lima CS: The flavonoid rutin modulates microglial/macrophage activation to a CD150/CD206 M2 phenotype. Chem Biol Interact 2017, 274:89-99.

60. Gao T, Chen Z, Chen H, Yuan H, Wang Y, Peng X, Wei C, Yang J, Xu C: Inhibition of HMGB1 mediates neuroprotection of traumatic brain injury by modulating the microglia/macrophage polarization. Biochem Biophys Res Commun 2018, 497:430-436.

61. Bu W, Ren H, Deng Y, Del MN, Guley NM, Moore BM, Honig MG, Reiner A: Mild Traumatic Brain Injury Produces Neuron Loss That Can Be Rescued by Modulating Microglial Activation Using a CB2 Receptor Inverse Agonist. Front Neurosci 2016, 10:449.

62. Kumar A, Barrett JP, Alvarez-Croda DM, Stoica BA, Faden Al, Loane DJ: NOX2 drives M1-like microglial/macrophage activation and neurodegeneration following experimental traumatic brain injury. Brain Behav Immun 2016, 58:291-309.

\section{Tables}

\section{Figures}



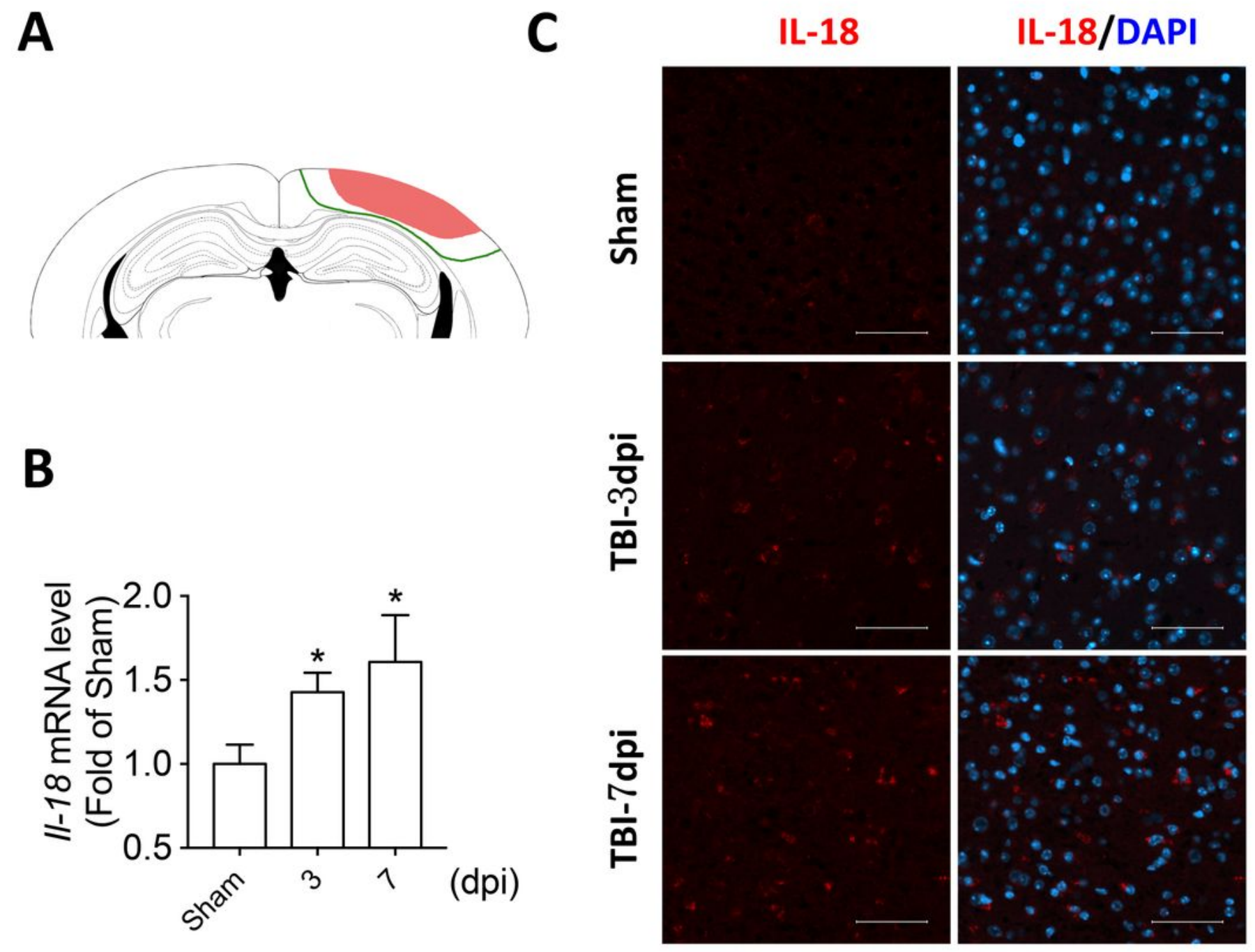

D

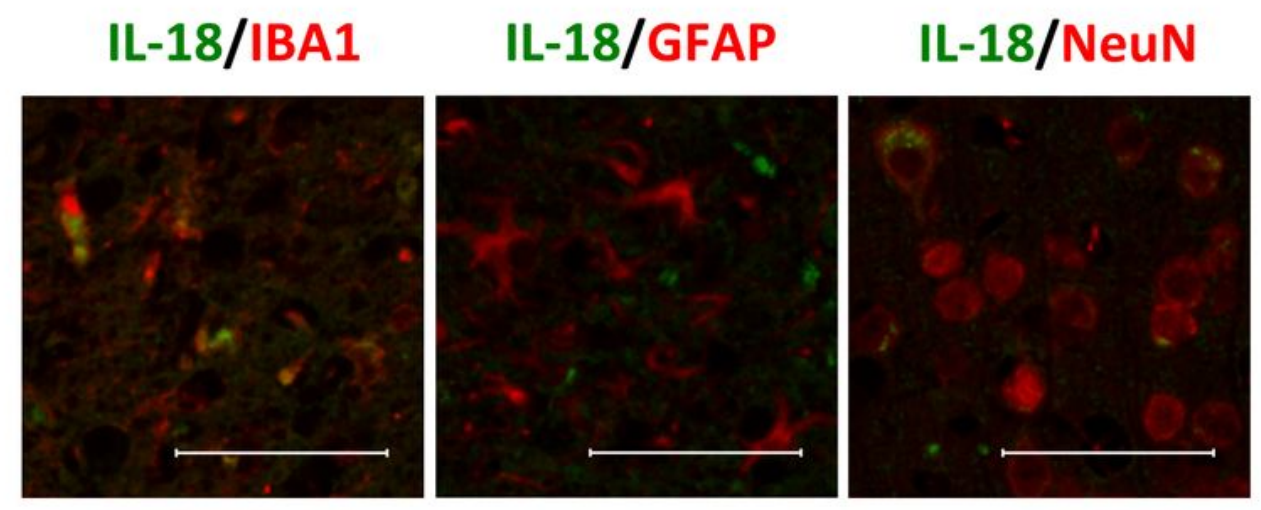

Figure 1

IL-18 expression was upregulated in the injured ipsilateral cortex after TBI (A) A schematic of a brain section after TBI. Areas in red refer to lesion sites, and areas depicted by green lines refer to the regions of interest. (B) The mRNA levels of II-18 in the injured ipsilateral cortex at 3 and 7 days after TBI evaluated by qRT-PCR. Data are presented as the mean \pm SD; ${ }^{*} p<0.05$ vs. the sham group, $\# p<0.05$ vs. WT mice ( $n$ = 6). (C) Representative immunofluorescence micrographs of the ipsilateral cortex after staining with an 
anti-IL-18 primary antibody at 3 and 7 days after TBI. Red fluorescence, IL18-positive cells. Nuclei were stained with DAPI (blue). Scale bar, $50 \mu \mathrm{m}$. (D) Representative fluorescence micrographs of the cortex at 7 days post TBI, illustrating colocalization of IL-18 (green) with IBA1/GFAP/NeuN (red). Scale bar, $50 \mu \mathrm{m}$.

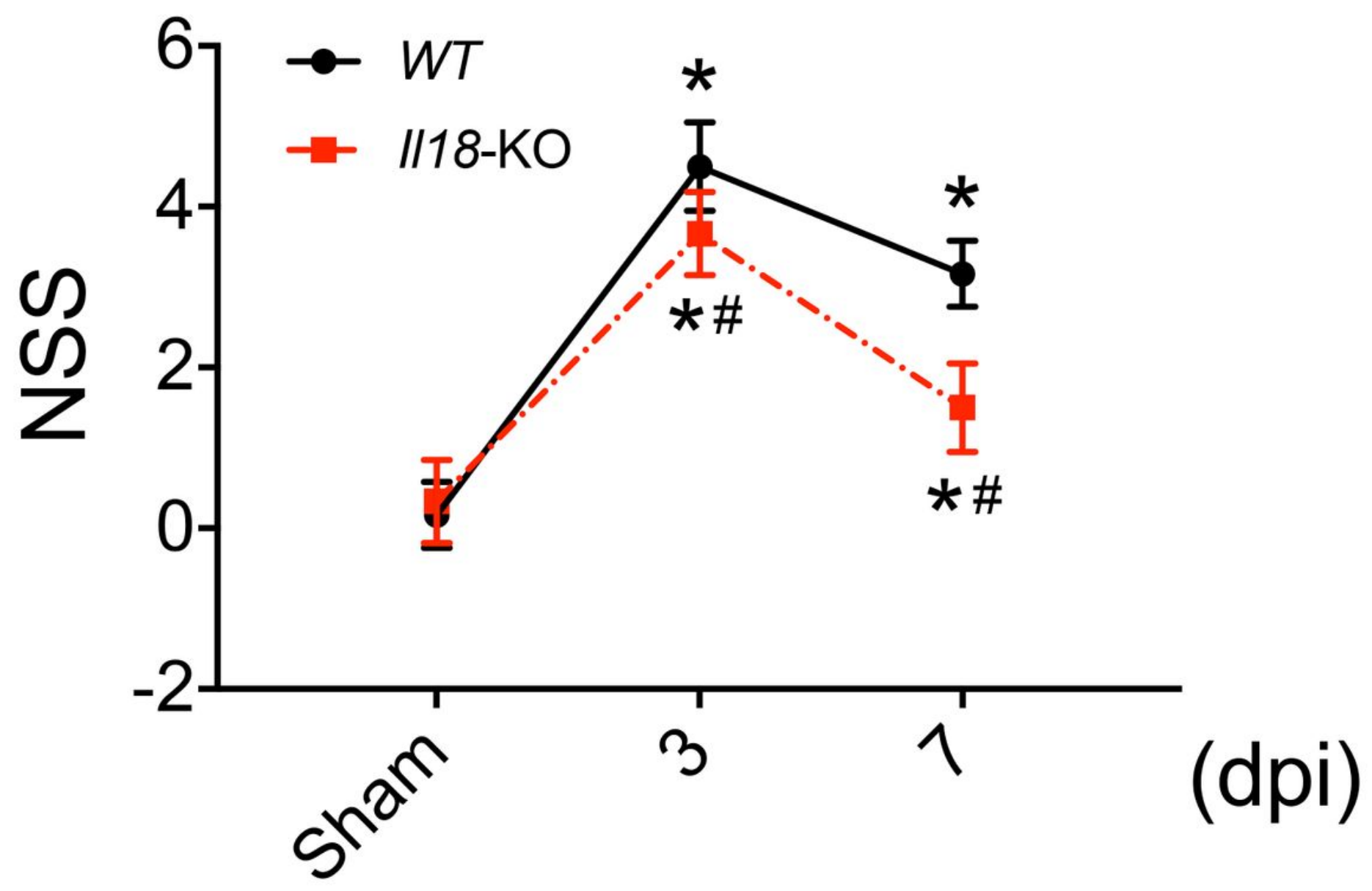

Figure 2

II-18 deficiency ameliorated neurological deficits after TBI A 10-point NSS was used to assess the role of IL-18 in neurological deficits. Data are presented as the mean $\pm S D ;{ }^{*} p<0.05$ vs. the sham group, $\# p<0.05$ vs. WT mice $(n=12)$. 


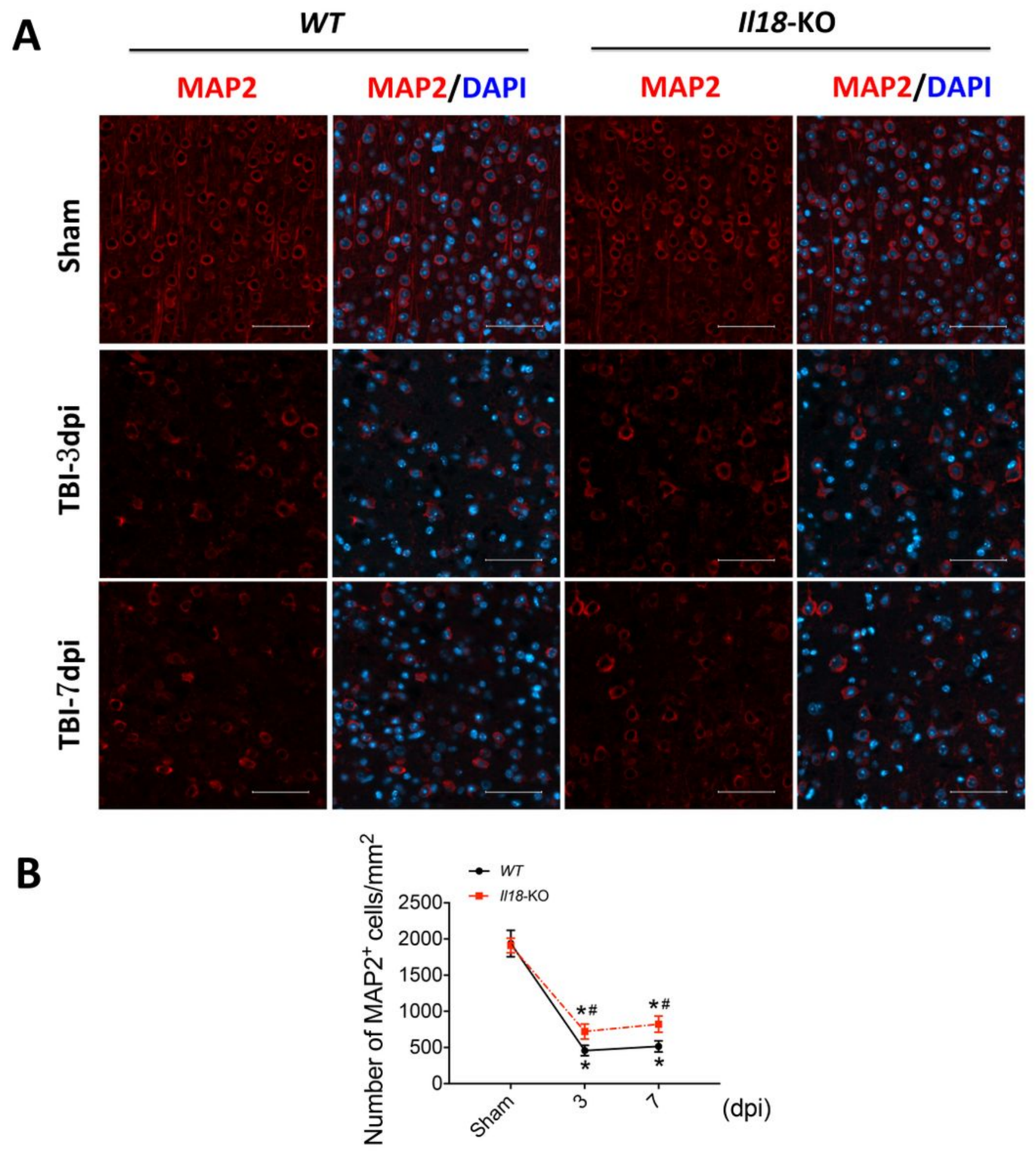

Figure 3

II-18 deficiency ameliorated neuron survival after TBI (A) Representative neuron-specific MAP2 immunofluorescence staining in pericontusional cortex sections at 3 and 7 days after TBI from WT or II18-KO mice. Nuclei were stained with DAPI (blue). Scale bar, $50 \mu \mathrm{m}$. (B) Corresponding quantification of the number of MAP2+ cells in $1 \mathrm{~mm} 2$ of tissue. Data are presented as the mean $\pm \mathrm{SD} ;{ }^{*} \mathrm{p}<0.05 \mathrm{vs}$. the sham group, $\# p<0.05$ vs. WT mice $(n=6)$. 


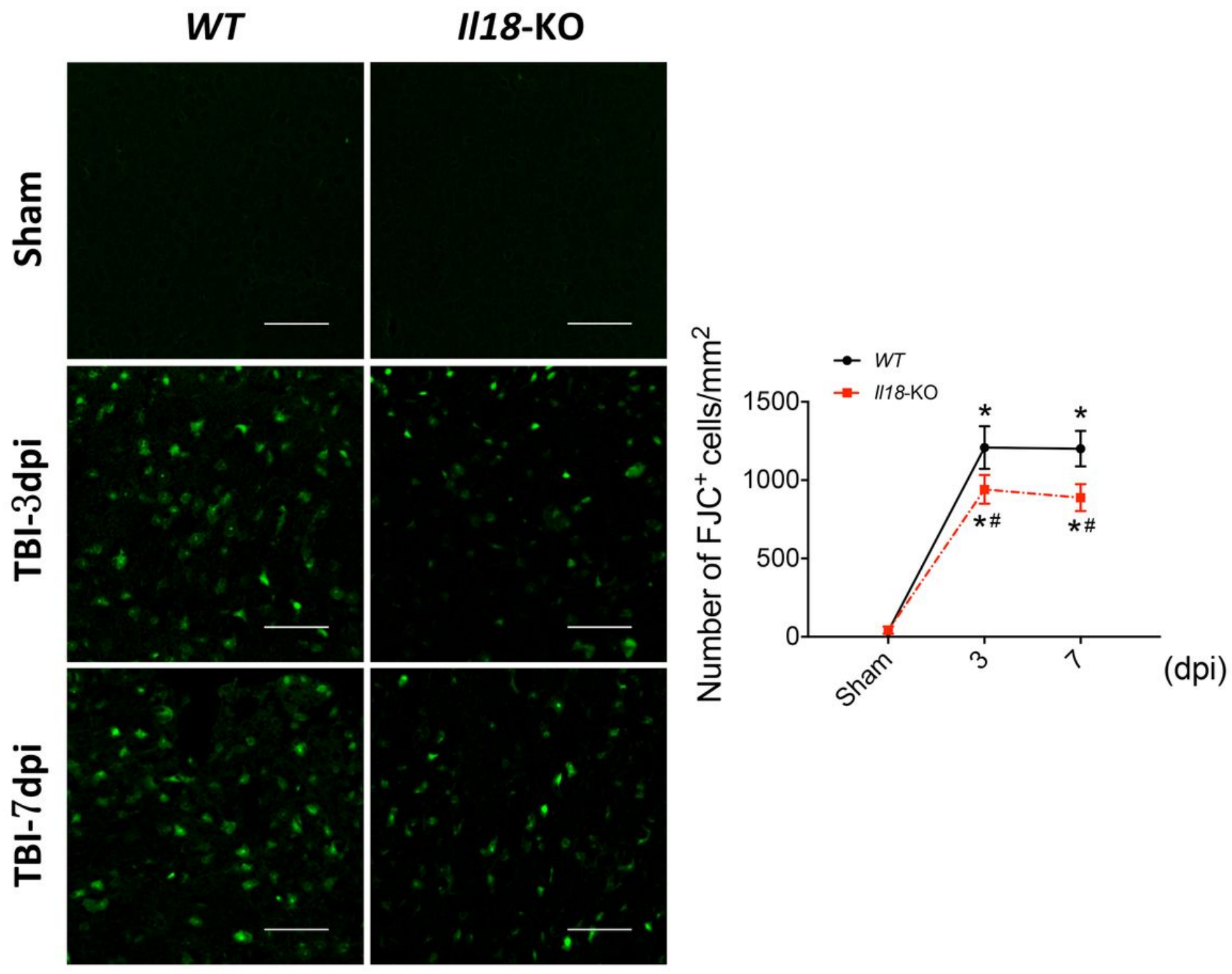

Figure 4

II-18 deficiency attenuated neurodegeneration after TBI Representative Fluoro-Jade C (FJC) staining in pericontusional cortex sections at 3 and 7 days after TBI from WT or II18-KO mice. Degenerated neuronal cells were labeled with green fluorescence. Corresponding quantification of the number of FJC+ cells in 1 $\mathrm{mm} 2$ of tissue (right panel). Data are presented as the mean $\pm \mathrm{SD} ;{ }^{*} \mathrm{p}<0.05 \mathrm{vs}$. the sham group, $\# \mathrm{p}<0.05$ vs. WT mice $(n=6)$. Scale bar, $50 \mu \mathrm{m}$. 
A

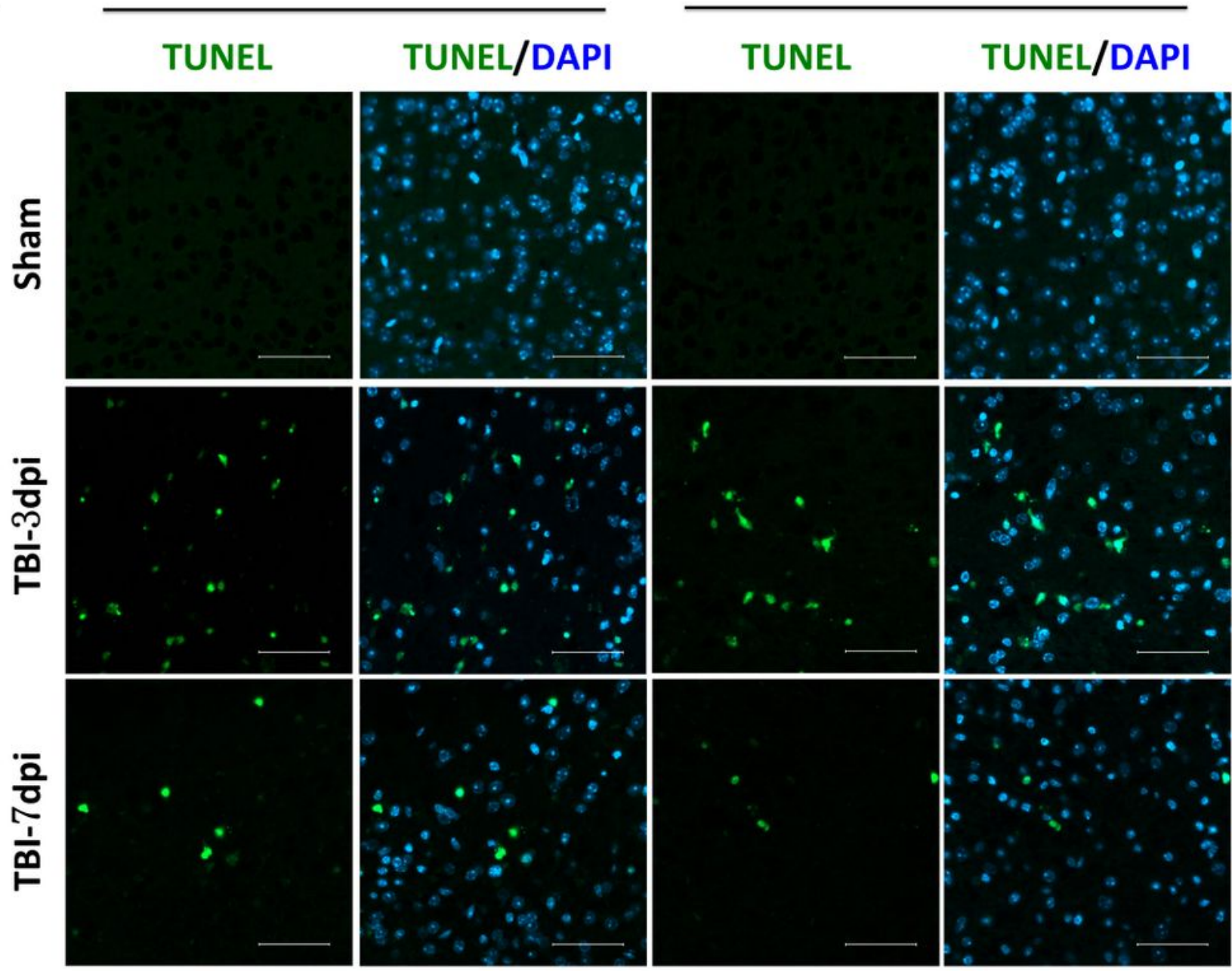

B

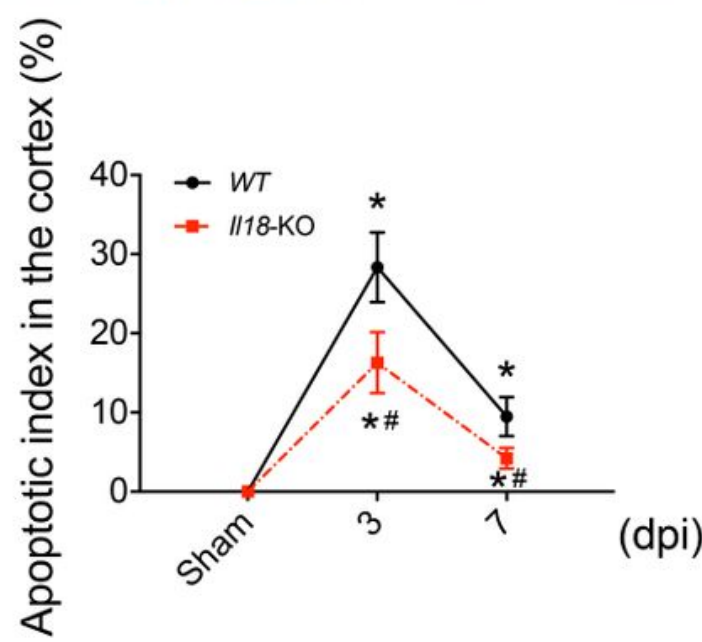

Figure 5

II-18 deficiency attenuated apoptotic cell death after TBI (A) Representative micrographs for TUNEL (green) and DAPI (blue) staining in the pericontusional site at 3 and 7 days after TBI. Scale bar, $50 \mu \mathrm{m}$. (B) The cell apoptotic index was calculated as the number of TUNEL+ nuclei divided by the total number of DAPI+ nuclei. Data are presented as the mean $\pm S D ;{ }^{*} p<0.05$ vs. the sham group, $\# p<0.05$ vs. WT mice $(n=6)$. 


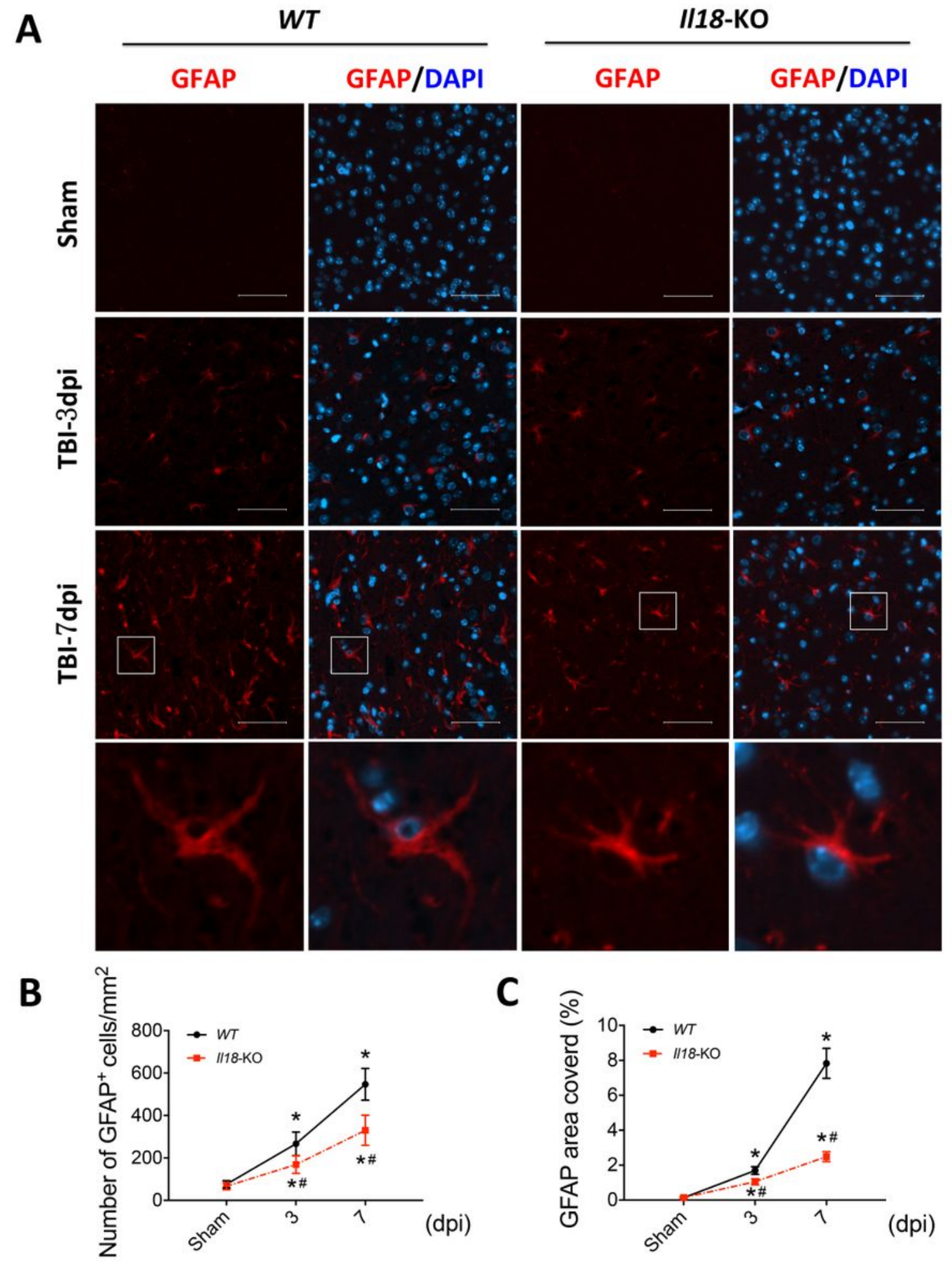

Figure 6

II-18 deficiency attenuated the TBI-induced activation of astrocytes (A) Representative astrocyte-specific GFAP immunofluorescence staining in pericontusional cortex sections at 3 and 7 days after TBI from WT or II18-KO mice. Nuclei were stained with DAPI (blue). Scale bar, $50 \mu \mathrm{m}$. (B) Corresponding quantification of the number of GFAP+ cells in $1 \mathrm{~mm} 2$ of tissue. Data are presented as the mean $\pm \mathrm{SD}$; ${ }^{*} p<0.05 \mathrm{vs}$. the 
sham group, $\# p<0.05$ vs. WT mice $(n=6)$. (C) Corresponding quantification of the percent area of GFAP+ cells. Data are presented as the mean \pm SD; ${ }^{*} p<0.05$ vs. the sham group, $\# p<0.05$ vs. WT mice $(n=6)$.

A

WT

IBA1
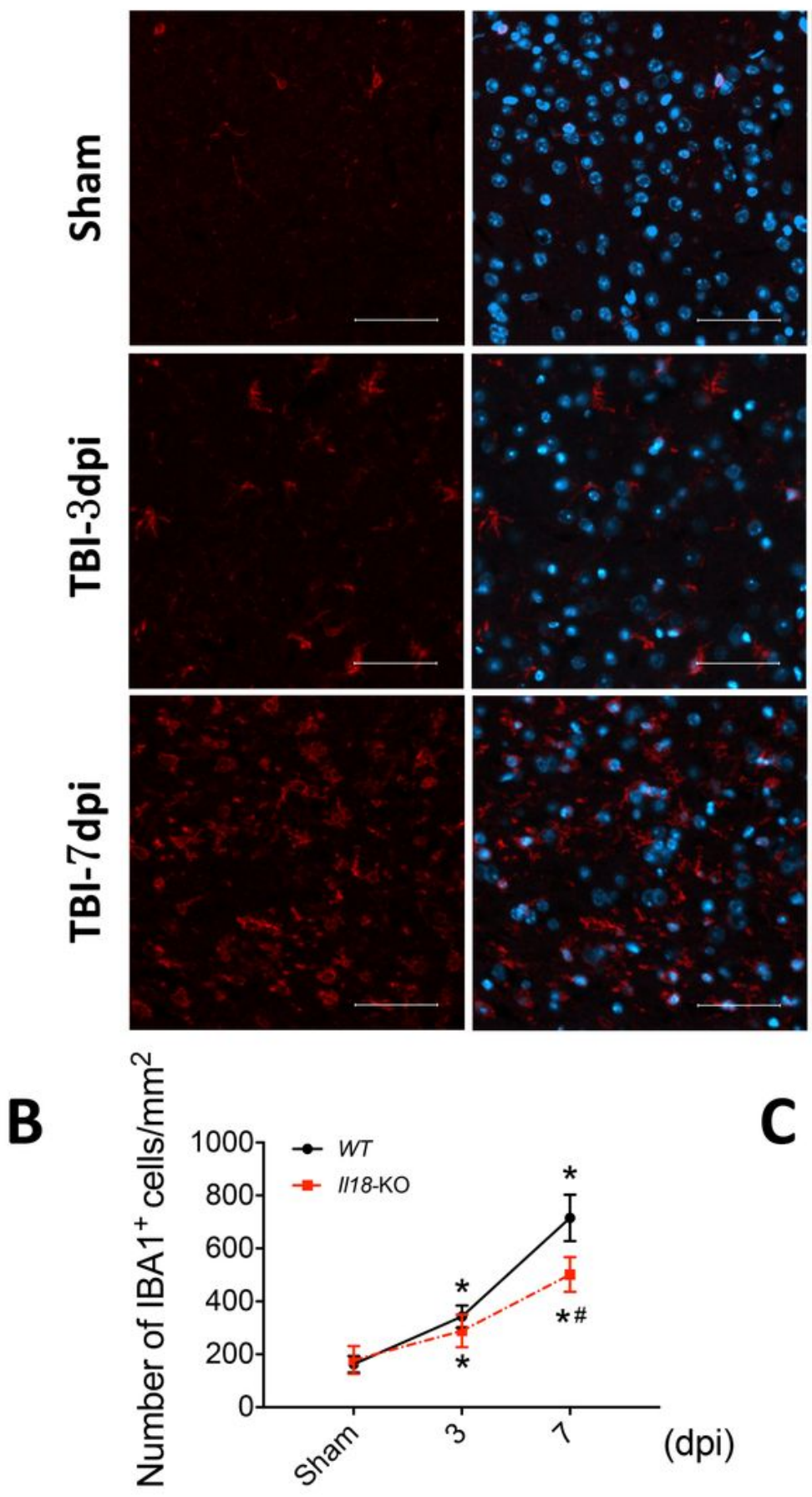
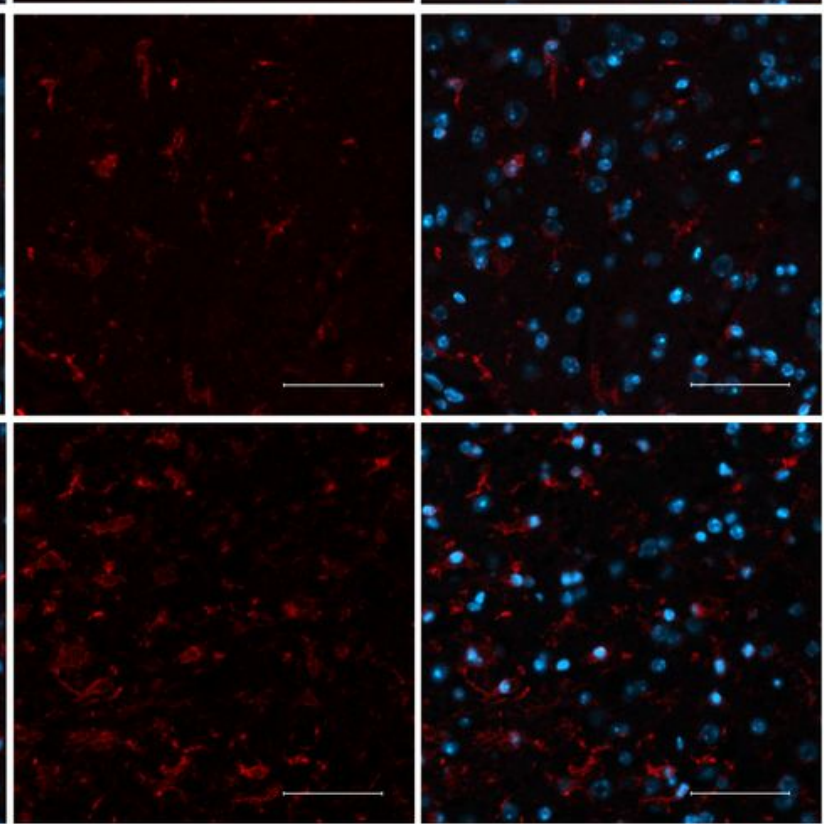

II18-KO

IBA1/DAPI
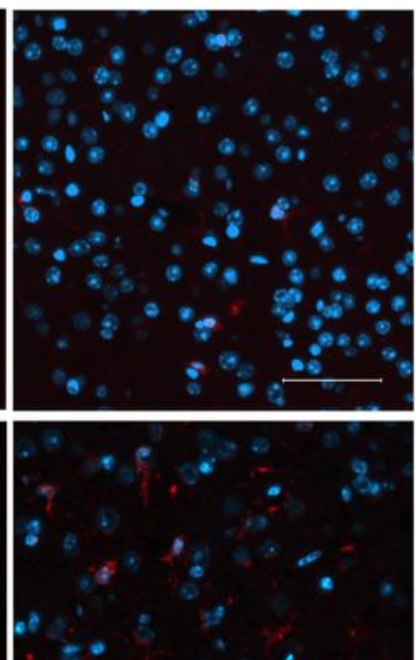

C

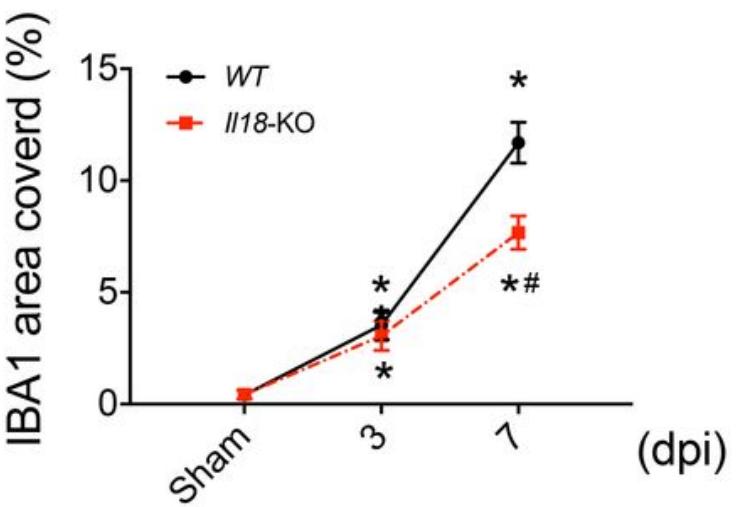

\section{Figure 7}

II-18 deficiency attenuated TBI-induced microglial activation (A) Representative microglia-specific IBA1 immunofluorescent staining in pericontusional cortex sections at 3 and 7 days after TBI from WT or II18$\mathrm{KO}$ mice. Nuclei were stained with DAPI (blue). Scale bar, $50 \mu \mathrm{m}$. (B) Corresponding quantification of the 
number of IBA1+ cells in $1 \mathrm{~mm} 2$ of tissue. Data are presented as the mean $\pm S D ;{ }^{*} p<0.05$ vs. the sham group, $\# p<0.05$ vs. WT mice $(n=6)$. (C) Corresponding quantification of the percent area of IBA1+ cells. Data are presented as the mean $\pm S D$; ${ }^{*} p<0.05$ vs. the sham group, $\# p<0.05$ vs. WT mice $(n=6)$.

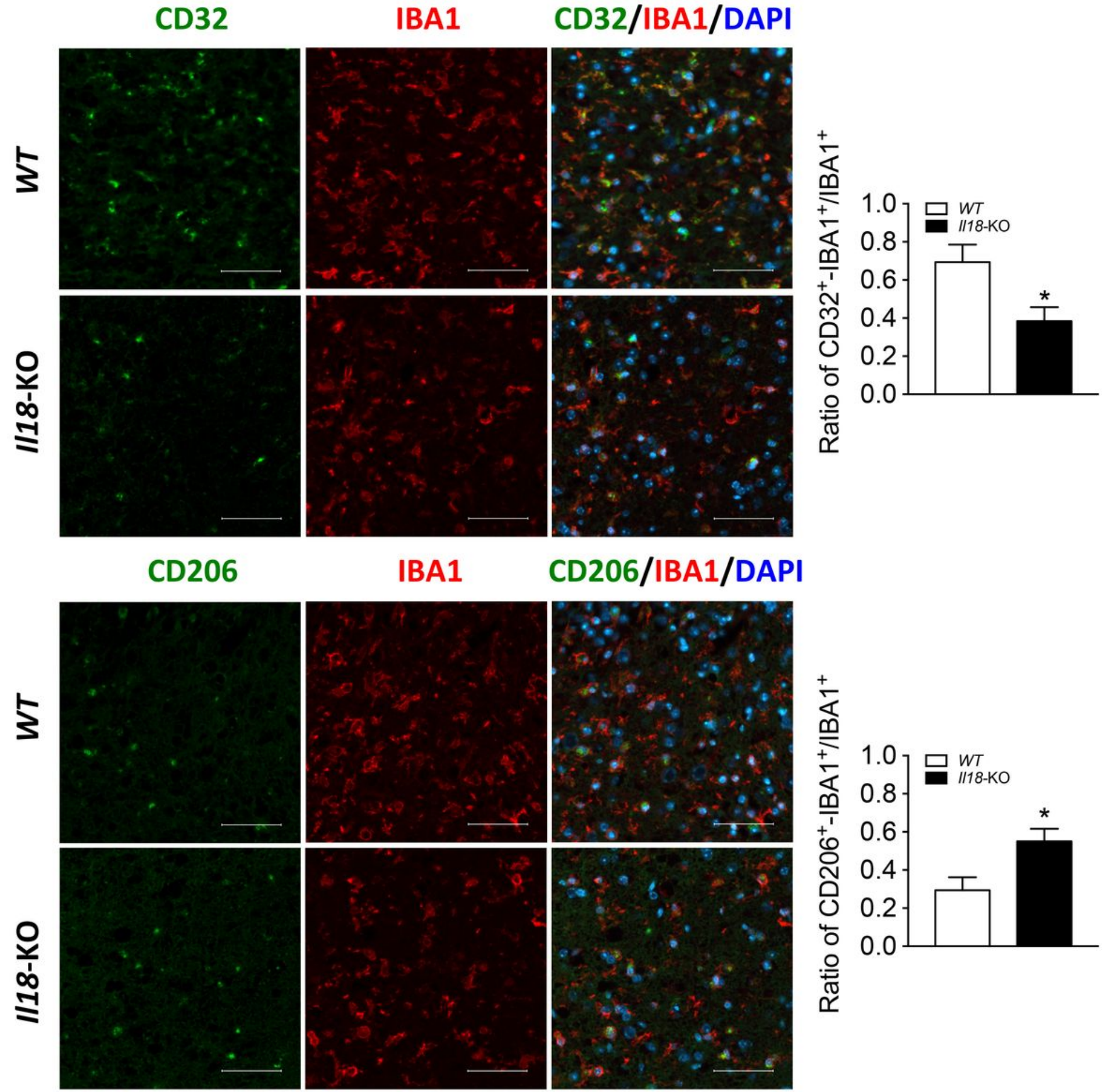

Figure 8

II-18 deficiency reduced the frequency of TBI-induced M1-like 'proinflammatory' microglia Representative immunofluorescence images of CD32 (green) and IBA1 (red) staining to identify M1-like microglia and CD206 (green) and IBA1 (red) staining to identify M2-like microglia in pericontusional cortex sections at 7 
dpi from WT or II18-KO mice. The percentages of CD32+IBA1+ and CD206+IBA1+ double-positive cells versus IBA1-positive cells were calculated, respectively. Data are presented as the mean $\pm \mathrm{SD} ;{ }^{*} \mathrm{p}<0.05 \mathrm{vs}$. the sham group, $\# p<0.05$ vs. WT mice $(n=6)$. Scale bar, $50 \mu m$.

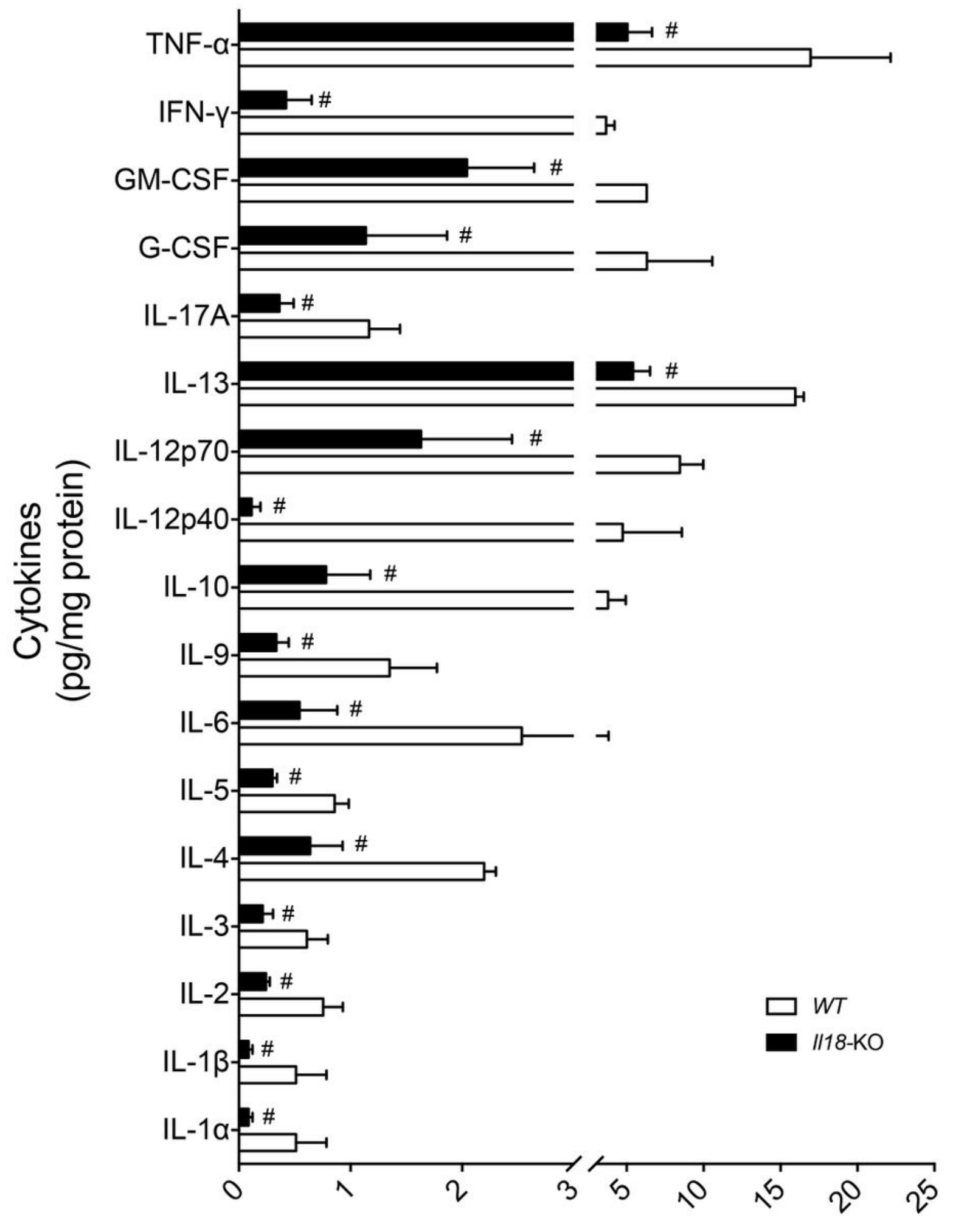

Figure 9

II-18 deficiency attenuated the TBI-induced secretion of cytokines The levels of cytokines in the injured ipsilateral cortex at 3 dpi were evaluated by a Luminex assay. Data are presented as the mean \pm SD; \#p< 
0.05 vs. WT mice $(n=6)$

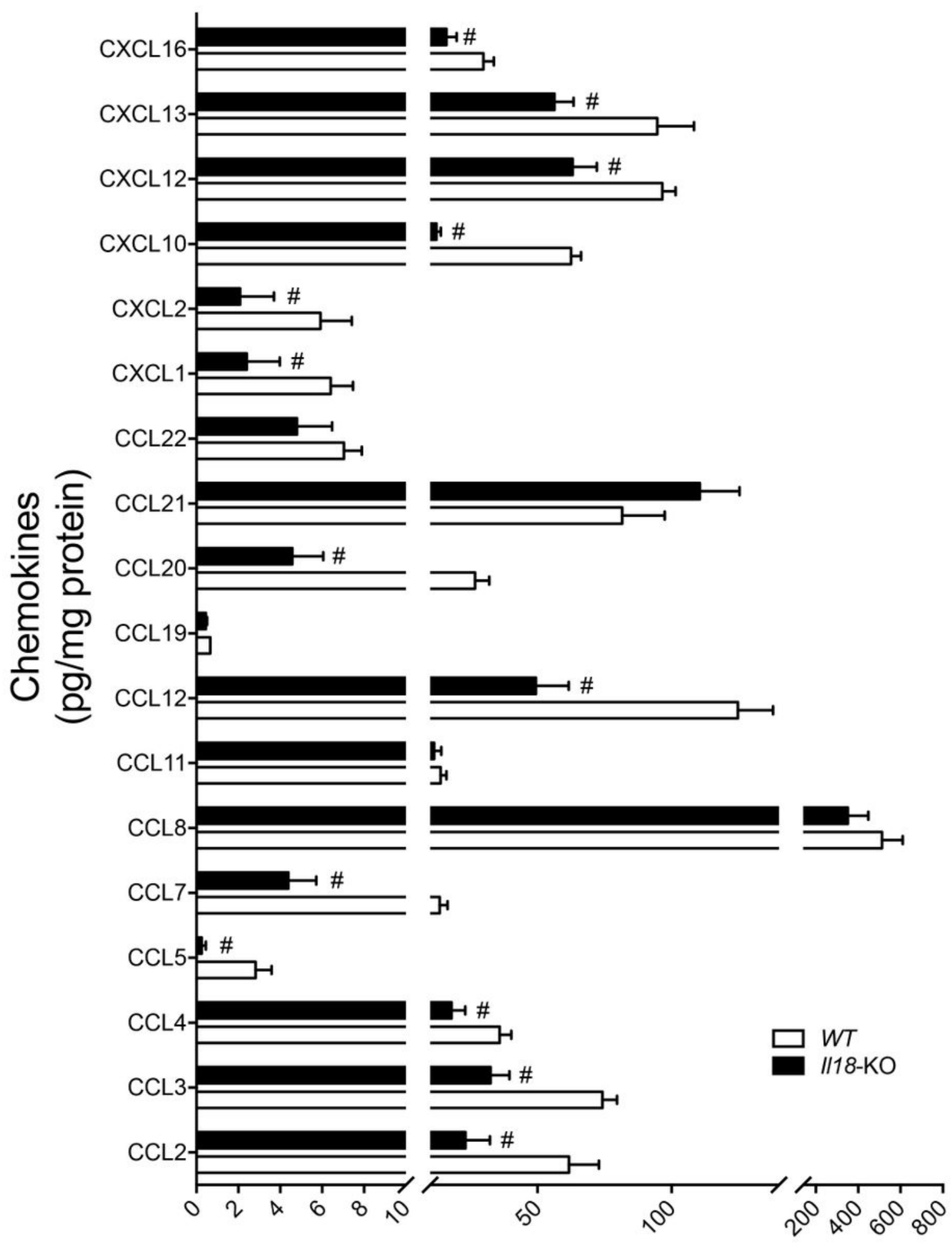

Figure 10

II-18 deficiency attenuated the TBI-induced secretion of chemokines The levels of chemokines in the injured ipsilateral cortex at $3 \mathrm{dpi}$ were evaluated by a Luminex assay. Data are presented as the mean \pm SD; \#p< 0.05 vs. WT mice $(n=6)$. 


\section{Supplementary Files}

This is a list of supplementary files associated with this preprint. Click to download.

- Additionalfile1.FigureS1.tif

- Additionalfile3.Figures3.tif

- Additionalfile4.Tables1.docx

- Additionalfile5.Tables2.docx

- Additionalfile2.Figures2.tif 